\title{
The Faintest and Closest Clusters in CFHTLS W4
}

\section{Mukadder İğdi Şen ${ }^{1 *}$}

\begin{abstract}
Using T0007 version of CFHTLS Wide 4 (W4) field observations, photometric redshift values were calculated for $0.2 \leq \mathrm{z} \leq 1.5$. Also the values of VIPERS spectroscopic catalog were used to control the obtained photometric redshifts. Using the galaxy SEDs in Le PHARE program the results obtained. The best results were found in $17.5 \leq \mathrm{i}_{\mathrm{AB}}^{\prime} \leq 21.5$ as catastrophic error of $8.5 \%$ and accuracy value of 0.031 . The catastrophic error and accuracy values in $17.5 \leq \mathrm{i}_{\mathrm{AB}}{ } \leq 24$ were determined as $4.1 \%$ and 0.030 respectively; the best correlation coefficient belongs to elliptical galaxies $(r=0.929)$. The relationship for elliptical galaxies between the photometric and spectroscopic redshift values was determined as $\mathrm{z}_{\mathrm{p}}=$ $(0.953 \pm 0.008) \mathrm{z}_{\mathrm{s}}+(0.083 \pm 0.014)$. The detected clusters are in the range of $0.475 \leq \mathrm{z}_{\mathrm{s}} \leq 0.937$. In our calculations made by taking the Hubble constant value of $71 \mathrm{~km} \mathrm{~s}^{-1} \mathrm{Mpc}^{-1}$, the distance of the faintest cluster of (i') $23.946 \mathrm{mag}$ is $1.22 \times 10^{23} \mathrm{~km}$ and the closest cluster is $0.61 \times 10^{23} \mathrm{~km}$.
\end{abstract}

Keywords: Photometric Redshift, Large Scale Structure, Galaxies, CFHTLS

\section{Introduction}

If a system is moving away from us, a redshift occurs. Photometric redshifts have been used to determine the large-scale structure of Universe in last times.

The spectral redshift method is giving quite accurate values. In this method, long observation times are needed to observe the faint objects away from us, therefore the wavelength of the spectral lines of the object can be found. However, taking long observation times from large telescopes are difficult. Thus, for this reason, photometric observation is used to obtain redshifts of many objects in a very short time. A lot of studies are performed to reduce the error of photometric redshifts.

The galaxies are also moving away from us. They have redshift values according to their distances, because the universe has expanding which called as "Hubble expansion". Because of this expansion, galaxies and other type objects such as stars and quasars have "the cosmological redshift". There is a relation between distancing speed and distances as seen in the Hubble Law's relation in (1):

$$
\mathrm{v}=\mathrm{H} \cdot \mathrm{d}
$$

$$
\frac{\Delta \lambda}{\lambda_{0}}=\frac{\mathrm{v}}{\mathrm{c}}
$$

Here, $\lambda_{0}$ is the value of wavelength when the object is stable, $\Delta \lambda=\lambda-\lambda_{0}$ is the shift amount of the spectral line, $\lambda$ is the value of observed wavelength, $\mathrm{c}$ is light speed and $\mathrm{v}$ is the speed of "coming and distancing".

${ }^{1}$ Trakya Üniversity, Edirne Vocational College of Technical Sciences, 22020, Edirne, TURKEY

*Correspondingauthor: mukaddersen@trakya.edu.tr
Citation (Atıf): İğdi Şen, M., (2018). The Faintest and Closest Clusters in CFHTLS W4Plants. Bilge International Journal of Science and Technology Research, 2 (special issue), 53-58. 
The position of the lines in a spectrum is related to the relative speed between the source and the observer, as seen from the Doppler relation (2) ( $\lambda_{0}$ and $\mathrm{c}$ are known, $\Delta \lambda$ is obtained from observations and thus the speed of $\mathrm{v}$ can be found and distance can be calculated by using Hubble constant value as $71 \mathrm{~km} \mathrm{~s}^{-1} \mathrm{Mpc}^{-1}$ (Feuerbacher and Stoewer, 2006)). The reliability of photometric redshifts depends on the method used to find the photometric redshifts. In the range of $1.5<\mathrm{z}<2.2$, star formation activity is maximum and the accumulation of general mass are present, but there is no galaxy-like structure, therefore this $\mathrm{z}$ range $(1.5<\mathrm{z}$ $<2.2)$ in the non-galactic astronomy is called by "redshift desert".

Arnouts et al. (1999) used the standard $\varkappa^{2}$ (chi-square) method. No spectral information was used in their work. They used elliptical, Sbc, Scd, and irregular galaxies SEDs observed by Coleman et al. (1980) and added a starburst (SB) SED from Kinney et al. (1996) and adapted them to ultraviolet and infrared wavelengths. No redness value was applied for the spectral types prior to Scd. The results of the standard $x^{2}$ method were compared with the values of spectroscopic redshift taken VIPERS for $i^{\prime} \leq 22.5$. In the faint magnitudes, galaxies of $\Delta z=\left(z_{s}-z_{p}\right)>1$ is very numerous often in the range of $1.5<z_{p}<3$. Most of the catastrophic errors are due to misidentification of Lyman and Balmer cuttings. It is stated that the infrared observation data is important in reducing of errors.

Ilbert et al. (2006) analyzed using the data of $i_{A B}^{\prime} \leq 24$ for the D1 deep area of CFHTLS. The authors used 3241 spectroscopic redshift values obtained from spectroscopic observations made by VIMOS (VVDS022604) for the D1 field in the range $0 \leq \mathrm{z} \leq 5$ to evaluate the photometric redshift results. It is stated by writers that galaxy types are important on the results. The results show that the best accuracy and catastrophic error values are in the range $0.2 \leq \mathrm{z} \leq 1.5$.

Coupon et al. (2009) obtained $z_{p}$ values by applying the filter fitting method to Terapix T0004 version for CFHTLS wide and deep fields. They used 16983 highly accurate $z_{\mathrm{S}}$ values from several spectroscopic surveys to test their $z_{p}$ results. According to the authors, the range of $0.2<z_{p}<1.5$ is better compatible with $\mathrm{z}_{\mathrm{s}}$ values. The accuracy value in $\mathrm{i}_{\mathrm{AB}}<24$ for the D1 field is 0.028 and the catastrophic error rate is $3.50 \%$. In large areas, the accuracy rate in $\mathrm{i}_{\mathrm{AB}}{ }_{\mathrm{BB}}<22.5$ is 0.036 and the catastrophic error rate is $2.80 \%$. The catastrophic error rates in large areas are 5\% in $\mathrm{i}_{\mathrm{AB}}^{\prime}<23$ and $10 \%$ in $\mathrm{i}_{\mathrm{AB}}^{\prime}<24$.

\section{Material and Method}

\subsection{Material}

The Wide 4 field (W4) photometric observations of the T0007 version made by Terapix with CFHTLS $u^{*} g^{\prime} r^{\prime} i ' z$ 'filters of Megacam cameras were used to obtain the best photometric refshifts. This data and the spectroscopic redshifts taken from VIPERS (VIMOS Public Extragalactic Redshift Survey) catalog which include the spectroscopic data of CFHTLS W4.

The central coordinates $(\alpha, \delta)$ (J2000) of CFHTLS Deep and Wide fields are given in Table 1 (Hudelot et al., 2012). The center coordinates of the W4 field are: RA (2000) = 221318 and DEC (2000) = +01 1900 . CFHTLS W4 field has 1258700 objects in the redshift range of $0<\mathrm{z}<6$. The observations of CFHTLS W4 in T0007 observation was divided into 25 subfields and their central coordinate informations (RA, DEC) are taken from Terapix 1 (2012) were given in Table 2 of İğdi-Şen (2018). 
Table 1. Central coordinates of CFHTLS wide (W1, W2, W3 and W4) and deep fields (D1, D2, D3 and D4) (Hudelot et al., 2012).

\begin{tabular}{ll}
\hline CFHTLS region & $\begin{array}{l}\text { Coordinates of Reference Center } \\
(\alpha, \delta)(\mathrm{J} 2000)\end{array}$ \\
\hline D1 & $02: 25: 59-04: 29: 40$ \\
D2 & $10: 00: 2802: 12: 30$ \\
D3 & $14: 19: 27+52: 40: 56$ \\
D4 & $22: 15: 31-17: 43: 56$ \\
W1 & $02: 18: 00-07: 00: 00$ \\
W2 & $08: 57: 49-03: 19: 00$ \\
W3 & $14: 17: 54+54: 30: 31$ \\
W4 & $22: 13: 18+01: 19: 00$ \\
\hline
\end{tabular}

W4_Spectro_PDR1 catalog of VIPERS (the Visible Multi-Object Spectrograph (VIMOS)) is used in our analyszes. This catalog is made to obtain the spectroscopic values of $\sim 100000$ galaxies for $\mathrm{i}^{\prime}{ }_{\mathrm{AB}}<22.5$ and $0.5<\mathrm{z}<1.2$. The numbers of observed, detected objects and their measured and reliable object numbers are seen in Table 2 (Garilli et al., 2014).

Table 2. The number informations of objects in W4_Spectro_PDR1 catalog (Garilli et al., 2014).

\begin{tabular}{lllll}
\hline Field & Observed objects & Detected objects & Measured redshift values & Reliable redshift values \\
\hline W4 & 31260 & 29897 & 28041 & 24050
\end{tabular}

\subsection{Method}

To determine the cluster members from the redshift distribution, the object in every redshift ( $\mathrm{z}$ ) is taken as if it is the center of a cluster. Limit range of $z \pm 1.44 \sigma_{\Delta z /(1+z s)}$ is selected as the limits of cluster. Here, the correlation of $\sigma_{\Delta z /(1+z s)}$ shows the accuracy value which is 1.44 (means $1 \sigma$ ) and it covers more than $85 \%$ of the cluster member galaxies. According to Wen and Han (2011), larger $\mathrm{z}$ range contains more galaxies, however, there are more galaxies in the background. For this reason, less bright galaxies with a limit of $M\left(i^{\prime}\right)<-21.5$ are excluded in cluster detection. For detection of galaxy clusters, the histogram of the $\mathrm{N}-\mathrm{z}_{\mathrm{s}}$ distributions are used. For $i^{\prime}{ }_{A B}<23$ and $i^{\prime}{ }_{A B}<24$ in $0<z<5$ redshift range, the histogram graphs of $N\left(z_{p}\right)-z_{p}$ and $N\left(z_{s}\right)-z_{s}$ obtained by combining the $z_{p}$ and $z_{s}$ values in steps of 0.05 are shown in Figure 1. The different spectroscopic distribution is found for each cluster. Since the cluster members are gravitationally connected, in the histograms, the majority of the cluster members must be at about in the same $\mathrm{z}$, with a broad peak that reflects the total mass of the cluster. Since the number of cluster members determines the total mass, this width depends on the mass of the cluster. If a galaxy with the greatest mass is in the potential center of the cluster, the z-value of the cluster equals the peak value. 

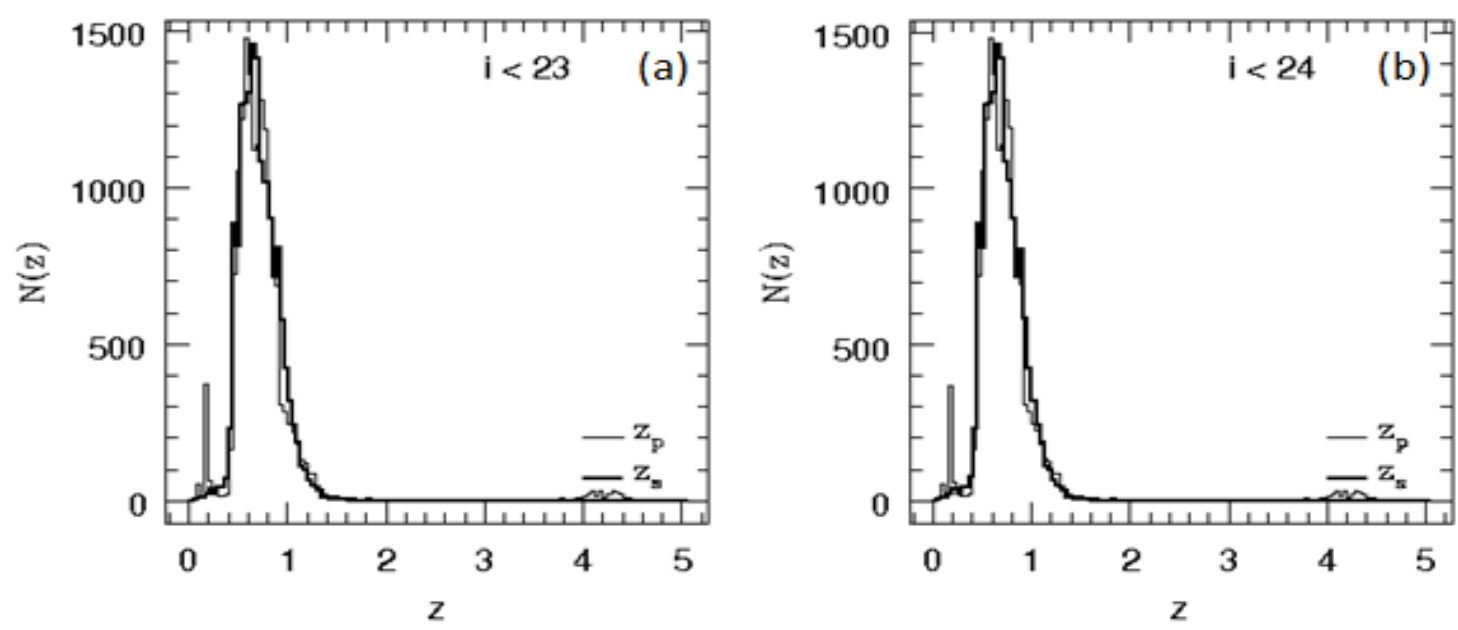

Figure 1. The graphs of $\mathrm{N}(\mathrm{z})$ - $\mathrm{z}$ number density drawn with $\mathrm{z}_{\mathrm{s}}$ values belong to spectroscopic observations and our $z_{p}$ values for a) $i_{A B}^{\prime}<23$ and b) $i_{A B}^{\prime}<24$ in $0<z_{p}<5$. $z_{s}$ is indicated by a thick solid line. The values of $z_{\mathrm{p}}$ and $z_{\mathrm{s}}$ are combined in steps of 0.05 .

If the compared second object is a member of the cluster, then the $z$-value is in the range of $z-1.44 \times 0.029$ $<\mathrm{z}<\mathrm{z}+1.44 \times 0.029$, and the distance between two objects is less than/or equal to $0.5 \mathrm{Mpc}$. Firstly, the distance of self-motion between the object and earth is calculated, then the angular diameter is calculated for the physical diameter taken as $0.5 \mathrm{Mpc}$ in redshift of the cluster. The galaxies located at these specific diameters are detected. For this purpose, for a cluster with diameter of $0.5 \mathrm{Mpc}$, the core region of $0.25 \mathrm{Mpc}$ radius (D0) and the galaxies within the ring (D1) between $0.5-1 \mathrm{Mpc}$ are detected (see Figure 2); the numbers of galaxies are calculated and the surface density ratios (D0 / D1) are found by dividing them into their circular areas $\left(\pi r^{2}\right)$.

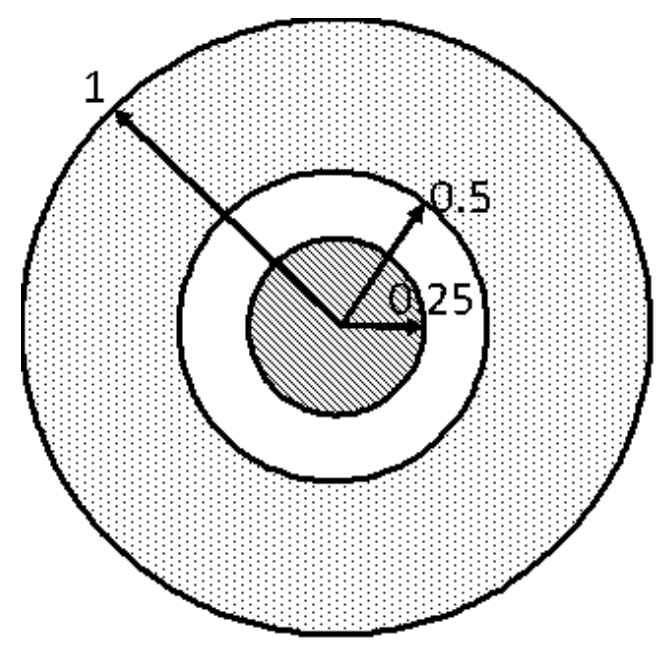

Figure 2. A sample of a core zone with a radius of $0.25 \mathrm{Mpc}$ and a ring of $0.5-1 \mathrm{Mpc}$ taken to identify galaxy clusters.

\section{Results}

In İğdi-Şen (2018), the obtaining methods of $\mathrm{z}_{\mathrm{p}}$ values in $0<\mathrm{z}<5$ were explained; the accuracy and catastrophic values between $z_{p}$ and $z_{s}$ for these SEDs to compare the magnitudes in five different $i^{\prime}{ }_{A B}$ ranges were given; obtained results from CFHTLS SEDs $\left(\eta=11.40 \%\right.$ and $\left.\sigma_{\Delta z /(1+z s)}=0.039\right)$ used in this study. 
The accuracy value $\left(\sigma_{\Delta z /(1+z s)}=0.029\right)$ in the correlation $\left(\mathrm{z} \pm 1.44 \sigma_{\Delta z /(1+z s)}\right)$ used to determine the cluster boundaries as taken from Ilbert and et al. (2006). When $\mathrm{M}\left(\mathrm{i}^{\prime}\right)<-21.5$ limit was set, the galaxy numbers in our catalog reduced from 28108 to 7164 . The informations of 55 clusters detected from these galaxies with $z_{s}$ valueand their coordinates are shown in Table 3.

Table 3. The properties of detected the faintest and the brightest clusters in CFHTLS W4. D0/D1 is the surface density rate of cluster.

\begin{tabular}{lllllll}
\hline $\begin{array}{l}\text { RA center } \\
\text { deg }\end{array}$ & $\begin{array}{l}\text { DEC center } \\
\text { deg }\end{array}$ & $\begin{array}{l}\text { Brightness } \\
i^{\prime}\end{array}$ & $z_{p}$ & $z_{s}$ & M(i') & D0/D1 \\
\hline 331.4277 & 2.1641 & 20.676 & 0.466 & 0,527 & -21.411 & 4.8 \\
333.6160 & 0.9046 & 23.946 & 0.770 & 0.935 & -19.292 & 8 \\
\hline
\end{tabular}

The distances of determined faintest and brightest galaxy clusters are calculated and given in Table 4.

Table 4. The distances of faintest and brightest galaxy clusters.

\begin{tabular}{ll}
\hline $\begin{array}{l}\text { The faintest cluster } \\
\left(\mathrm{i}^{\prime}{ }_{\mathrm{AB}}=23.946 \mathrm{mag}\right)\end{array}$ & $\begin{array}{l}\text { The brightest cluster } \\
\left(\mathrm{i}^{\prime}{ }_{\mathrm{AB}}=20.676 \mathrm{mag}\right)\end{array}$ \\
\hline $3.95 \times 10^{6} \mathrm{kpc}$ & $2.23 \times 10^{6} \mathrm{kpc}$ \\
\hline
\end{tabular}

\section{Discussion and Conclusions}

The obtained values of accuracy (0.030) and catastrophic error $(1.92 \%)$ for 5214 galaxies at $17.5 \leq \mathrm{i}$ 'AB $\leq$ 21.5 and $0.4 \leq \mathrm{zp} \leq 1$ (see Table 6 in İğdi-Şen, 2018) are the best results. The obtained results from elliptical galaxies are $\sigma \Delta \mathrm{z} /(1+\mathrm{zs}) \sim 0.030$ and $\eta \sim 4.10 \%$ (see Table 7 in İğdi-Şen (2018)). The best correlation coefficient belongs to elliptical galaxies $(r=0.929)$. The best value of the linear fit equation between galaxy types belongs to the elliptical galaxies. Here, $z p=(0.953 \pm 0.008) \mathrm{zs}+(0.083 \pm 0.014)$ is obtained. The fact that the coefficient of zs is close to 1 suggests a good correlation between zp and zs. (see Figure 5 in İğdi-Şen (2018)). The apparent brightness is a key parameter for the photometric redshift estimations as seen in Table 6 of İğdi-Şen (2018).

According to the rates of cluster surface density seen in Table 3, the rates of surface density of cluster members are quite high. This situation shows that the selection method of cluster members is correct because cluster members should have a higher surface density inside of the cluster area in the small radial circular region of our calculations. The detected galaxy clusters are in the range of $0.475 \leq \mathrm{zs} \leq 0.937$. The faintest cluster is $i^{\prime} A B=23.946$ mag and the brightest cluster is $i^{\prime} A B=20.676$ mag. The distance of faintest cluster is $3.95 \times 106 \mathrm{kpc}(1.22 \times 1023 \mathrm{~km})$. The brightest cluster was discovered in $2.23 \times 106 \mathrm{kpc}$. The closest cluster is in $2.01 \times 106 \mathrm{kpc}(0.61 \times 1023 \mathrm{~km})$.

\section{Acknowledgements}

Thanks to Yüksel Karataş for his advisory support and to Christophe Benoist for all the scientific contributions and guidance during this study.

\section{References}

Arnouts, S., Cristiani, S., Moscardini, L., Matarrese, S., Lucchin, F., Fontana, A., Giallongo, E. (1999). Measuring and modelling the redshift evolution of clustering: the Hubble Deep Field North. Monthly Notices of the Royal Astronomical Society, 310(2), 540-556.

Coleman, G. D., Wu, C. C., Weedman, D. W. (1980). Colors and magnitudes predicted for high redshift galaxies. The Astrophysical Journal Supplement Series, 43, 393-416. 
Coupon, J., Ilbert, O., Kilbinger, M., McCracken, H. J., Mellier, Y., Arnouts, S., ... , Le Brun, V. (2009). Photometric redshifts for the CFHTLS T0004 deep and wide fields. Astronomy \& Astrophysics, 500(3), 981-998.

Feuerbacher, B. and Stoewer, H., (2006). Utilization of Space: Today and Tomorrow, Springer, ISBN: $354029970 X$.

Garilli, B., Guzzo, L., Scodeggio, M., Bolzonella, M., Abbas, U., Adami, C., ..., Cappi, A. (2014). The VIMOS Public Extragalactic Survey (VIPERS)-First Data Release of 57204 spectroscopic measurements. Astronomy \& Astrophysics, 562, A23.

Hudelot, P., Goranova, Y., Mellier, Y., McCracken, H. J., Magnard, F., Monnerville, M., ..., Betoule, M. (2012). T0007: The Final CFHTLS Release. [visiting date: 1st June 2013].

Ilbert, O., Arnouts, S., McCracken, H. J., Bolzonella, M., Bertin, E., Le Fevre, O., ..., Tresse, L. (2006). Accurate photometric redshifts for the CFHT legacy survey calibrated using the VIMOS VLT deep survey. Astronomy \& Astrophysics, 457(3), 841-856.

İğdi-Şen, M. (2018). The Reliability of Redshift Values in Determination of Structures in the Universe, JoCREST, 4, 69-80.

Kinney, A. L., Calzetti, D., Bohlin, R. C., McQuade, K., Storchi-Bergmann, T.,Schmitt, H. R. (1996). Template ultraviolet to near-infrared spectra of star-forming galaxies and their application to kcorrections. The astrophysical journal. Chicago. Vol. 467, no. 1, pt. 1 (Aug. 1996), p. 38-60.

Le Fèvre, O., Vettolani, G., Garilli, B., Tresse, L., Bottini, D. B. V. L., Le Brun, V., ..., Zanichelli, A. (2005). The VIMOS VLT deep survey-First epoch VVDS-deep survey: 11564 spectra with $17.5 \leq$ I \$ Itextit $\{$ Itextbf $\{$ |small $\mathrm{AB}\}\} \$ \leq 24$, and the redshift distribution over $0 \leq \mathrm{z} \leq 5$. Astronomy \& Astrophysics, 439(3), 845-862.

Terapix1, (2012). CFHTLS-Deep \&Wide targets, Terapix, http://terapix.iap.fr/ cplt/T0007/table_syn_T0007.html, [visiting date: 1st January 2015].

Wen, Z. L.and Han, J. L. (2011). Galaxy Clusters at High Redshift and Evolution of Brightest Cluster Galaxies, The Astrophysics of Journal, 734, 68W. 